\title{
Validation of Assessment Categories of the Patient-generated Subjective Global Assessment Tool in Classifying the Nutritional Status of Cancer Patients
}

\author{
Lohith Mysuru Shivanna, Namratha Pai Kotebagilu, Asna Urooj \\ Department of Studies in Food Science and Nutrition, University of Mysore, Mysuru, Karnataka, India
}

Abstract

Background: The patient-generated-subjective global assessment (PG-SGA) tool is considered as the most appropriate nutrition screening tool in oncological setting, since the scoring system enables the patients to be triaged for nutrition intervention. In this study, an attempt was made to determine the precision in classifying patients with cancer as per the Subjective global assessment (SGA) rating or Nutritional Triage recommendations using the PG-SGA tool. Methods: The study was conducted in cancer hospitals of Mysore, Karnataka, India, to recruit 136 cancer patients receiving treatment during a period of 1 year. The PG-SGA tool version 3.22 .15 was used for eliciting information and to assess the nutritional status of cancer patients. Results: Among 136 cancer patients, 95.74\%, 97.05\%, and 74.54\% of head-and-neck cancers (HNC), gastrointestinal cancers (GIC), and other cancers (OC), respectively, were malnourished (SGA B + C) according to the SGA categories (SGAC), whereas $93.61 \%, 97.05 \%$, and $81.81 \%$ of $\mathrm{HNC}$, GIC, and $\mathrm{OC}$, respectively, were categorized under severe nutrition risk as per the scored nutritional triage recommendations (SNTR). SGAC had an area under curve (AUC) of 0.961 , whereas nutritional triage recommendations had an AUC of 1.0, signifying higher precision of using SNTR to assess the nutritional risk and need for nutritional intervention among cancer patients. Conclusions: Overall, SNTR is more precise in assessing the nutritional status among cancer patients than SGAC and also helps in developing a better nutritional care plan for effective prognosis.

Keywords: Cancer, food intake, gastrointestinal cancers, head-and-neck cancers, nutritional status, patient-generated subjective global assessment, subjective global assessment, weight loss

\section{INTRODUCTION}

Cancer is a complex, multifactorial disease involving abnormal cell growth and development of malignant tumors. According to the GLOBOCAN report, 2012, the 5-year prevalence of cancer in India was found to be 665,000 in men and $1,126,000$ in women. ${ }^{[1]}$ The World Health Organization: Non-communicable diseases Country profile 2014 has documented the mortality rate of 7\% for cancers for all ages and both the genders in India. ${ }^{[2]}$ Cancer is associated with malnutrition mainly due to disease-induced and treatment-induced complications. Conventional treatment for cancer such as radiotherapy and chemotherapy induces side effects which interfere with food intake and worsen the nutritional status of cancer patients. Weight loss during chemotherapy or radiotherapy can diminish the safety and effectiveness of the treatment. Inadequate food intake along with cancer cachexia can elevate the weight loss

\begin{tabular}{|l|l|}
\hline & Access this article online \\
\hline Quick Response Code: & Website: \\
\hline & www.ijnpnd.com \\
\hline
\end{tabular}

trajectory. Nutritional intake deficits may occur early and will become more severe with disease progression. ${ }^{[3]}$ Therefore, assessment of nutritional status before planning any treatment schedule becomes vital to reduce the rate of disease catabolism. Nutritional status can be assessed with various anthropometric indices such as height, weight, body mass index (BMI), mid-upper arm circumference, and skinfold thickness; biochemical parameters such as hemoglobin, serum albumin, creatinine, urea, complete blood count, white blood cells, and clinical observation of the fat, muscle, and fluid status; and dietary data using $24 \mathrm{~h}$ diet recall and food frequency.

Address for correspondence: Dr. Asna Urooj, Department of studies in Food Science and Nutrition, University of Mysore, Manasagangotri, Mysuru - 570 006, Karnataka, India. E-mail: asnaurooj@foodsci.uni-mysore.ac.in

This is an open access journal, and articles are distributed under the terms of the Creative Commons Attribution-NonCommercial-ShareAlike 4.0 License, which allows others to remix, tweak, and build upon the work non-commercially, as long as appropriate credit is given and the new creations are licensed under the identical terms.

For reprints contact: reprints@medknow.com

How to cite this article: Shivanna LM, Kotebagilu NP, Urooj A. Validation of assessment categories of the patient-generated subjective global assessment tool in classifying the nutritional status of cancer patients. Int $\mathrm{J}$ Nutr Pharmacol Neurol Dis 2018;8:79-85. 
Among the various tools used to assess the nutritional status, patient-generated subjective global assessment (PG-SGA) tool is used worldwide. It has two sections: a patient-completed section which includes data regarding weight history, symptoms, dietary intake, and activity level and; a section completed by the health-care professional, who evaluates metabolic demand, considers disease in relation to nutritional requirements, and incorporates a physical assessment. ${ }^{[4]}$ The aim of the study was to determine the precision between SNTR and SGAC in classifying the nutritional status or the degree of malnutrition in cancer patients using the PG-SGA tool.

\section{Methods}

\section{Data collection}

A descriptive cross-sectional study was conducted to recruit 152 cancer patients attending cancer hospitals situated in Mysuru city, namely, Krishna Rajendra Hospital, Preethi Cancer Hospital, and HCG-Bharath Hospital and Institute of Oncology for treatment by adopting purposive sampling method between the months of September 2014 and November 2015. Permission was obtained to use the PG-SGA tool v3.22.15. ${ }^{[5]}$ Out of 152 participants, 16 could not complete the PG-SGA data, and hence 136 participants were included in the study. Permission from the Institutional Human Ethics Committee (IHEC-UOM No. 45 Res/2014-2015 dated August 07, 2014) and the respective hospitals were obtained. Participants with all types of histopathologically confirmed cancer undergoing chemotherapy/radiotherapy were recruited based on their willingness to participate and informed consent was obtained from all the participants. However, participants with critical illness or on enteral or parenteral feed were excluded from the study. The PG-SGA data was obtained by researchers experienced in conducting nutritional assessment of cancer patients. Each patient was classified as well nourished (SGA A), moderately or suspected of being malnourished (SGA B), or severely malnourished (SGA C) as per SGAC. In addition, they were also grouped as well nourished (SGA A) and malnourished (SGA B $+\mathrm{C}$ ). The PG-SGA total score was calculated using data on weight history, symptoms, dietary intake, activity level, metabolic stress, disease category, and physical examination. Based on the PG-SGA score, each patient was classified as severe nutrition risk (SNR) (PG-SGA score $>$ ) and no/mild nutrition risk (NMNR) (PG-SGA score 0-8) group as per SNTR. The cut-off point of a PG-SGA score $\geq 9$ is routinely used to indicate a critical need for improved symptom management and/or nutrition intervention options. ${ }^{[6,7]}$

\section{Data analysis}

The data were analyzed using statistical tool SPSS version 16.0 (SPSS Inc., Chicago, IL, USA).

Descriptive statistics were reported as mean \pm standard deviation, medians, interquartile ranges, frequencies, and percent. Data on food intake of past month were compared with weight loss in past 2 weeks using cross tabulation.
The relationship between PG-SGA score and BMI was determined by correlation analysis. A contingency table was used to determine sensitivity and specificity values of the PG-SGA compared to SGA and to validate its use to analyze the nutritional risk. Sensitivity and specificity values were calculated using the formulae,

a. Sensitivity $=\mathrm{TP} /(\mathrm{TP}+\mathrm{FN})$

b. $\quad$ Specificity $=\mathrm{TN} /(\mathrm{TN}+\mathrm{FP})$

Where, TP - true positive, FP - false positive, FN - false negative and $\mathrm{TN}$ - true negative.

The independent $t$-test was used to evaluate the difference in means among normally distributed variables, namely, malnourished and well-nourished patients as classified under SGA rating (age, weight, BMI, total PG-SGA score, and metabolic stress score); the nonparametric Mann-Whitney U-test was used to evaluate the difference in medians among variables without normal distribution as classified under both SGAC (Nutrition Impact Symptom [NIS] score, food intake score, weight loss score, activities, and function score) and SNTR (all variables with $P$ value). Receiver operating characteristic (ROC) curves were generated for the PG-SGA tool. It was used to test the accuracy of PG-SGA tool in discriminating between malnourished and well-nourished patients as classified by SGAC and also, patients who are at nutrition risk and not at nutrition risk as classified by SNTR. An AUC of 1.0 discriminates perfectly between well nourished and malnourished, whereas an AUC of 0.5 suggests an equal chance that a nutritional parameter will correctly identify patients who are nutritionally at risk. ${ }^{[8]}$ Therefore, the overall accuracy of the test would be higher when the ROC curve is exceptionally close to the upper left corner of the curve. ${ }^{[9]}$

\section{RESULTS}

Among the 136 participants, 47 (34.55\%), 34 (25\%), and 55 (40.44\%) were head-and-neck cancers (HNC), gastrointestinal cancers (GIC), and other cancers (OC), respectively.

The frequencies of NIS among various cancers are reported in Table 1. Among HNC patients, the most frequently observed NIS was dry mouth $(70.21 \%)$, followed by changes in taste perception (65.95\%) and dysphagia (65.95\%).

The frequency of nausea $(61.76 \%)$, dry mouth $(58.82 \%)$, and early satiety (58.82\%) was higher in GIC. Symptoms in OC followed the trend, pain $(45.45 \%)>$ nausea $(40 \%)>$ changes in taste perception $(40 \%)>$ early satiety $(40 \%)$. A higher percentage of subjects with OC $(41.81 \%)$ had no problem in eating. However, the severity of eating problems was the highest in HNC followed by GIC.

The frequencies of functional status among various cancers are reported in Table 2. The activity was affected more in HNC followed by GIC and OC, suggesting their inability in engaging usual activities. 
The results of food intake of past month and weight in past 2 weeks are given in Table 3 . Food intake of past month was compared with weight in past 2 weeks among 126 participants using the PG-SGA tool. It was observed that when the food intake in the past month remained unchanged, the weight also remained constant. There was no weight loss observed in participants who ate food more than the usual amount and weight gain was observed in six participants who ate more than usual. It can be observed that increased food intake will meet the energy demand and initiate anabolism, leading to better tolerance toward chemotherapy or radiotherapy regimen and improvement in the nutritional status. Majority of the participants $(n=99)$ had decreased food intake and weight loss in 2 weeks, as observed in $69(54.76 \%)$ participants. On the other hand, when the food intake in the past month was less than usual, there was an increase in weight among $6(4.76 \%)$

\begin{tabular}{|c|c|c|c|}
\hline NIS $(n=136)^{\mathrm{a}}$ & $\begin{array}{c}\text { HNC }(n=47) \\
n(\%)\end{array}$ & $\begin{array}{c}\text { GIC }(n=34), \\
n(\%)\end{array}$ & $\begin{array}{c}\text { OC }(n=55), \\
n(\%)\end{array}$ \\
\hline No problems eating & $3(6.38)$ & $10(29.41)$ & $23(41.81)$ \\
\hline No appetite & $13(27.65)$ & $14(41.17)$ & $17(30.90)$ \\
\hline Nausea & $22(46.80)$ & $21(61.76)$ & $22(40)$ \\
\hline Constipation & $10(21.27)$ & $13(38.23)$ & $18(32.72)$ \\
\hline Mouth sores & $20(42.55)$ & $3(8.82)$ & $3(5.45)$ \\
\hline Things taste funny & $31(65.95)$ & $18(52.94)$ & $22(40)$ \\
\hline Problems swallowing & $31(65.95)$ & $12(35.29)$ & $3(5.45)$ \\
\hline Pain & $29(61.70)$ & $18(52.94)$ & $25(45.45)$ \\
\hline Vomiting & $20(42.55)$ & $9(26.47)$ & $19(34.54)$ \\
\hline Diarrhea & $9(19.14)$ & $12(35.29)$ & $4(7.27)$ \\
\hline Dry mouth & $33(70.21)$ & $20(58.82)$ & $20(36.36)$ \\
\hline Smells bother me & $18(38.29)$ & $11(32.35)$ & $18(32.72)$ \\
\hline Feel full quickly & $26(55.31)$ & $20(58.82)$ & $22(40)$ \\
\hline
\end{tabular}

participants and weight remained constant in 24 (19.04\%) participants. This could be due to recent change in the dietary habits. Furthermore, the presence of edema can incorrectly interpret the actual weight.

The results of the PG-SGA score and the SGAC are given in Table 4 . Among the 136 participants, 119 participants $(87.5 \%)$ were malnourished falling under the SGA-B or SGA-C category implying moderate-to-severe malnutrition and 17 participants $(12.5 \%)$ were well nourished. According to the PG-SGA score, 122 participants $(89.70 \%)$ were at nutrition risk and required nutrition intervention and only 14 participants $(10.30 \%)$ were not at nutrition risk.

It can be observed that 117 participants were correctly classified as malnourished (true positive) and 12 participants correctly classified as well nourished (true negative), whereas 2 participants were misclassified as malnourished (false negative) and 5 participants were misclassified as well nourished (false positive). The results of data analyzed had sensitivity value of $0.983 \%$ or $98.3 \%$ which implies accuracy in identifying the true positive, i.e., identifying and classifying participants at nutrition risk having moderate or severe malnutrition. The specificity value was $0.705 \%$ or $70.5 \%$ which implies accuracy in identifying true negatives, i.e., participants who are not at nutrition risk and who are well nourished.

Clinical parameters according to SGAC are shown in Table 5. Among 136 cancer patients, 95.74\%, 97.05\%, and 74.54\% of $\mathrm{HNC}$, GIC, and $\mathrm{OC}$, respectively, were malnourished. Well-nourished participants were found to be diagnosed with OC (25.45\%), HNC (4.25\%), and GIC (2.94\%). Therefore, a higher degree of malnutrition is observed in HNC and GIC due to compromised oral cavity and gastrointestinal system, which affects food intake.

Malnourished patients were also significantly older $(P=0.040)$ compared to well-nourished participants. Parameters such as weight and BMI were significantly lower $(P<0.001)$ in

Table 2: Frequency of functional status among cancer patients

\begin{tabular}{lccc}
\hline Activities and function $(\boldsymbol{n = 1 3 6 )}$ & HNC $(\boldsymbol{n = 4 7 ) , \boldsymbol { n } ( \boldsymbol { \% } )}$ & GIC $(\boldsymbol{n}=\mathbf{3 4}), \boldsymbol{n}(\mathbf{\%})$ & $\mathbf{O C}(\boldsymbol{n}=\mathbf{5 5}), \boldsymbol{n}(\mathbf{\%})$ \\
\hline Normal with no limitations & $3(6.38)$ & $1(2.94)$ & $9(16.36)$ \\
Not my normal self, but fairly normal activities & $18(38.29)$ & $20(58.82)$ & $27(49.09)$ \\
Not feeling up to most things, in bed/chair $<$ half day & $19(40.42)$ & $6(17.64)$ & $10(18.18)$ \\
Little activity, most of the day in bed/chair & $7(14.89)$ & $7(20.58)$ & $9(16.36)$ \\
Pretty much bedridden & 0 & 0 & 0 \\
\hline
\end{tabular}

HNC: Head and neck cancers; GIC: Gastrointestinal cancers; OC: Other cancers

Table 3: Comparison of food intake in past month and weight in past 2 weeks among cancer patients

\begin{tabular}{|c|c|c|c|c|}
\hline \multirow{2}{*}{$\begin{array}{l}\text { Food intake } \\
\text { Past month }\end{array}$} & \multicolumn{4}{|c|}{ Weight in past 2 weeks $(n=126)$} \\
\hline & Decreased, $n$ (\%) & Not changed, $\boldsymbol{n}(\%)$ & Increased, $n$ (\%) & Total, $n(\%)$ \\
\hline Unchanged & $7(5.5)$ & $10(7.93)$ & $1(0.79)$ & $18(14.28)$ \\
\hline More than usual & 0 & $3(2.38)$ & $6(4.76)$ & $9(7.14)$ \\
\hline Less than usual & $69(54.76)$ & $24(19.04)$ & $6(4.76)$ & $99(78.57)$ \\
\hline Total & $76(60.31)$ & $37(29.36)$ & $13(10.31)$ & $126(100)$ \\
\hline
\end{tabular}


Table 4: Classification of cancer patients according to the patient-generated subjective global assessment score and subjective global assessment

\begin{tabular}{|c|c|c|c|}
\hline \multirow{2}{*}{$\begin{array}{l}\text { Nutrition risk } \\
\text { PG-SGA }\end{array}$} & \multicolumn{3}{|c|}{ SGA categories } \\
\hline & Malnourished (SGA B + C) & Well nourished (SGA A) & Total \\
\hline At nutrition risk (PG-SGA score $\geq 9$ ) & $117(\mathrm{TP})$ & $5(\mathrm{FP})$ & 122 \\
\hline Not at nutrition risk (PG-SGA score $0-8$ ) & $2(\mathrm{FN})$ & $12(\mathrm{TN})$ & 14 \\
\hline Total & 119 & 17 & 136 \\
\hline
\end{tabular}

TP: True positive; FP: False positive; FN: False negative; TN: True negative; $n=136$. SGA: Subjective global assessment; PG-SGA: Patient-generated SGA

Table 5: Clinical parameters for cancer patients as classified by subjective global assessment categories

\begin{tabular}{|c|c|c|c|c|}
\hline & Malnourished (SGA B + C) & Well nourished (SGA A) & Mean difference & $P$ \\
\hline Total (male/female), $n$ & $119(76 / 43)$ & $17(4 / 13)$ & - & - \\
\hline $\mathrm{HNC}, n(\%)$ & $45(95.74)$ & $2(4.25)$ & - & - \\
\hline GIC, $n(\%)$ & $33(97.05)$ & $1(2.94)$ & - & - \\
\hline OC, $n(\%)$ & $41(74.54)$ & $14(25.45)$ & - & - \\
\hline Age (years) $(n=136)$ & $55.97 \pm 12.91^{\mathrm{a}}$ & $48.65 \pm 17.82$ & 7.31 & $0.040^{\mathrm{b}}$ \\
\hline Weight $(\mathrm{kg})(n=133)$ & $53.78 \pm 10.96$ & $66.67 \pm 18.40$ & -12.89 & $<0.001$ \\
\hline $\operatorname{BMI}\left(\mathrm{kg} / \mathrm{m}^{2}\right)(n=133)$ & $21.02 \pm 4.63$ & $25.87 \pm 5.52$ & -4.84 & $<0.001$ \\
\hline Total PG-SGA score $(n=136)$ & $20.81 \pm 6.18$ & $8.24 \pm 3.52$ & 12.57 & $<0.001$ \\
\hline NIS score $(n=136)$ & $10.0(6.0,12.0)^{\mathrm{c}}$ & $1.0(0.0,4.0)$ & - & $<0.001^{\mathrm{d}}$ \\
\hline Food intake score $(n=136)$ & $2.0(1.0,3.0)$ & $0.0(0.0,1.0)$ & - & $<0.001$ \\
\hline Weight loss score $(n=136)$ & $1.0(0.0,3.0)$ & $0.0(0.0,0.0)$ & - & $<0.001$ \\
\hline Activities and function score $(n=136)$ & $1.0(1.0,2.0)$ & $0.0(0.0,1.0)$ & - & $<0.001$ \\
\hline Metabolic stress score $(n=136)$ & $3.40 \pm 0.96$ & $3.06 \pm 0.42$ & 0.34 & 0.015 \\
\hline
\end{tabular}

${ }^{\mathrm{a}}$ Mean \pm SD (all such values), ${ }^{\mathrm{b}}$ Independent $t$-test, ${ }^{\mathrm{c}}$ Median; $25^{\text {th }}$ and $75^{\text {th }}$ percentile in brackets (all such values), ${ }^{\mathrm{d}}$ Mann-Whitney U-test. HNC: Head and neck cancers; GIC: Gastrointestinal cancers; OC: Other cancers; BMI: Body mass index; SGA: Subjective global assessment; PG-SGA: Patient generated-SGA; NIS: Nutrition impact symptoms; SD: Standard deviation

malnourished group. The correlation of PG-SGA score and BMI $(r=-0.474, P<0.001)$ had statistical significance with expected negative correlation, i.e., the higher the PG-SGA score, lower is the BMI. NISs were higher and were significantly affected $(P<0.001)$ with a lower nutritional status. The scores of food intake, weight loss, activity, and function were significantly higher $(P<0.001)$ in malnourished patients than well nourished, indicating the influence of food intake on weight, activity, and function. Metabolic stress was also significantly higher $(P=0.015)$ in the malnourished group. Overall, the total PG-SGA score was significantly higher $(P<0.001)$ among malnourished patients.

ROC curve for SGAC is shown in Figure 1 and the PG-SGA tool classified as per SGAC had AUC $=0.961$, indicating near to perfect discrimination between malnourished and well-nourished group.

Clinical parameters according to SNTR are shown in Table 6. In this study, patients who were in critical need of nutrition intervention were classified as SNR group and patients who might/might not have required any nutrition intervention were classified as NMNR group. Results showed that $93.61 \%$ of HNC, $97.05 \%$ of GIC, and $81.81 \%$ of OC were classified under the SNR group. The need for nutrition intervention was higher in GIC followed by HNC and OC. In general, $89.7 \%$ of cancer patients required immediate nutrition intervention or critical emphasis on improved symptom management.

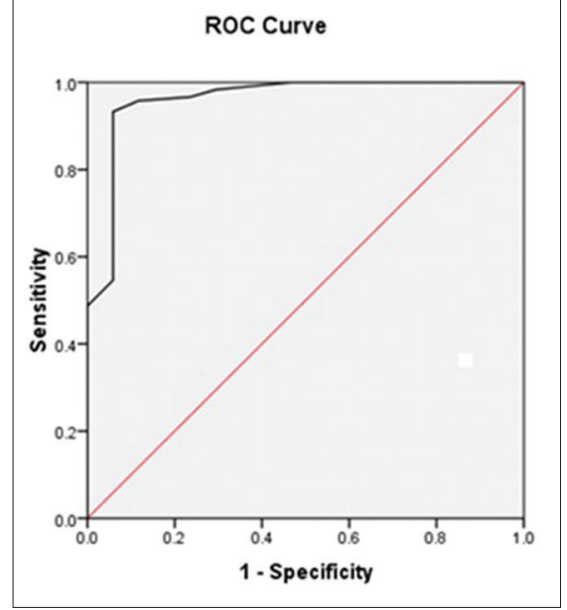

Figure 1: Receiver operating characteristic curves for subjective global assessment categories $(n=136)$. The reference line represents a curve for a receiver operating characteristic area of 0.5 . The area under the curve is 0.961

The nutrition risk was lower in OC (18.18\%). Patients with SNR had significantly lower weight $(P=0.009)$ and BMI $(P=0.020)$ than the NMNR counterpart. The results between patients with SNR and NMNR were comparable to malnourished and well-nourished parameters. The scores of food intake, weight loss, activity, and function were significantly higher $(P<0.001)$ in SNR group than NMNR 
Shivanna, et al.: Validation of assessment categories of the PG-SGA tool

\begin{tabular}{|c|c|c|c|}
\hline & Severe nutrition risk (PG-SGA Score $>9$ ) & No/mild nutrition risk (PG-SGA Score 0-8) & $P$ \\
\hline Total patients (male/female), $n$ & $122(76 / 46)$ & $14(4 / 10)$ & - \\
\hline $\mathrm{HNC}, n(\%)$ & $44(93.61)$ & $3(6.38)$ & - \\
\hline GIC, $n(\%)$ & $33(97.05)$ & $1(2.94)$ & - \\
\hline OC, $n(\%)$ & $45(81.81)$ & $10(18.18)$ & - \\
\hline Age (years) $(n=133)$ & $57.0(48.0,65.0)^{\mathrm{a}}$ & $52.5(44.7,61.2)$ & $0.34^{\mathrm{b}}$ \\
\hline Weight $(\mathrm{kg})(n=133)$ & $53.0(45.0,62.0)$ & $63.0(55.2,81.0)$ & 0.009 \\
\hline $\operatorname{BMI}\left(\mathrm{kg} / \mathrm{m}^{2}\right)(n=133)$ & $20.8(17.4,24.0)$ & $23.0(21.0,27.7)$ & 0.020 \\
\hline Total PG-SGA score $(n=136)$ & $20.0(16.0,26.0)$ & $7.0(6.0,8.0)$ & $<0.001$ \\
\hline NIS score $(n=136)$ & $9.0(6.0,12.0)$ & $0.0(0.0,1.0)$ & $<0.001$ \\
\hline Food intake score $(n=136)$ & $2.0(1.0,3.0)$ & $0.0(0.0,0.2)$ & $<0.001$ \\
\hline Weight loss score $(n=136)$ & $1.0(0.0,3.0)$ & $0.0(0.0,0.2)$ & 0.001 \\
\hline Activities and function score $(n=136)$ & $1.0(1.0,2.0)$ & $0.0(0.0,1.0)$ & $<0.001$ \\
\hline Metabolic stress score $(n=136)$ & $3.0(3.0,3.0)$ & $3.0(3.0,3.0)$ & 0.080 \\
\hline
\end{tabular}

${ }^{\mathrm{a}}$ Median; $25^{\text {th }}$ and $75^{\text {th }}$ percentile in brackets (all such values), ${ }^{\mathrm{b}}$ Mann-Whitney U-test. HNC: Head and neck cancers; GIC: Gastrointestinal cancers;

OC: Other cancers; BMI: Body mass index; SGA: Subjective global assessment; PG-SGA: Patient generated-SGA; NIS: Nutrition impact symptoms

group. However, metabolic stress was present in both the groups without any significant difference $(P=0.080)$. Overall, the total PG-SGA score was significantly higher $(P<0.001)$ among SNR group.

ROC curve for SNTR is shown in Figure 2, and the PG-SGA tool classified as per SNTR had AUC $=1.0$. Based on the AUC results, the overall accuracy of PG-SGA tool classified as per SNTR is higher and effective when compared to PG-SGA tool classified as per SGAC. This negligible dissimilarity might be due to the subjective approach while classifying SGAC.

\section{Discussion}

NIS is present in cancer patients at presentation before the initiation of treatment. A study conducted on HNC patients before treatment reported NIS, namely, pain and dysphagia as the most common symptoms among study population and dysphagia was regarded as the principal cause for reduced dietary intake leading to weight loss. If NIS, which influence dietary intake, is assessed and managed before/during the treatment, the involuntary weight loss can be prevented. ${ }^{[10]}$ The presence of pain and discomfort in cancer patients triggers stress response as a physiological effect and increases catabolism. It stimulates the release of catecholamines that alter gastrointestinal activity and decreases food intake. ${ }^{[1]}$ The severity of symptoms can be prevented by controlling the pain which would enhance the dietary intake in patients with terminal and advanced cancer, as it revamps their food intake even during anorexic symptoms. ${ }^{[12,13]}$ In a study conducted by Omlin et al. among 52 cancer patients, the most frequent NIS were smell alterations, constipation, abdominal pain, dysphagia, and epigastric pain. ${ }^{[14]}$

Functional status as measured by the activity level is affected in cancer patients due to the disease, presence of NIS, and decreased food intake. Literature reports that $47 \%$ of $\mathrm{HNC}$ patients have reduced functional capacity. ${ }^{[10]}$

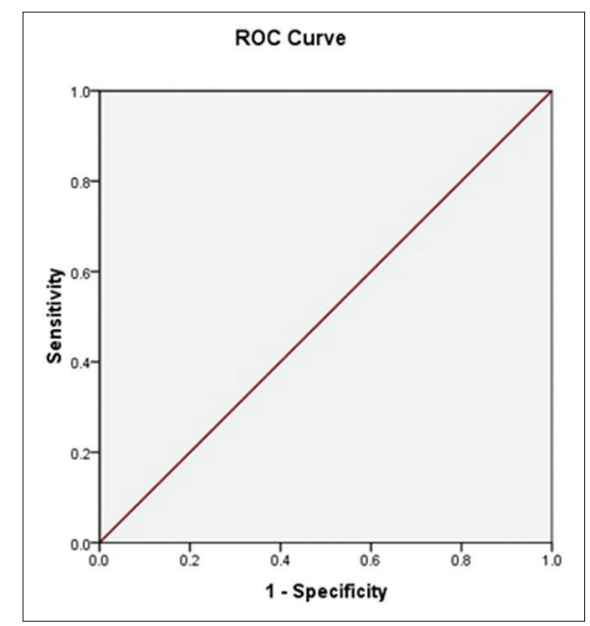

Figure 2: Receiver operating characteristic curves for scored nutritional triage recommendation $(n=136)$. The reference line represents a curve for a receiver operating characteristic area of 0.5 . The area under the curve is 1.0

Weight loss trajectory is crucial in cancer. In our study, few participants $(n=7)$ reported weight loss though the food intake in the past month remained unchanged, which could be due to increased catabolism, increased energy demand by the body, tumor-host competition for nutrients, or stage of cancer. ${ }^{[15]}$ The prognosis in cancer patients is determined by three factors, i.e., weight loss, reduced food intake, and systemic inflammation such as edema which gives a false body weight. ${ }^{[16]}$

The results of sensitivity and specificity of PG-SGA score of our study were comparable with reported studies wherein the PG-SGA score had a sensitivity of $98 \%$ and a specificity of $82 \%$ at predicting SGA classification. ${ }^{[17]}$ Gabrielson et al. also reported $97 \%$ sensitivity and $86 \%$ specificity for the PG-SGA score. ${ }^{[18]}$

Literature reports the presence of higher proportion of malnutrition among participants with lung cancer (100\%) and 
GIC (78\%); $;^{[18]}$ however, in our study, HNC patients exhibited a higher degree of malnourishment.

Gabrielson et al. reported that malnourished patients were significantly older $(P<0.001)$ and had lower BMI $(P<0.01)^{[18]}$ which was also observed in our study.

A study conducted by Bauer et al. also reported a correlation between PG-SGA score and BMI without statistical significance. ${ }^{[17]}$

The results of ROC curve for SGAC were similar to literatures reported by Gabrielson et al. (AUC $=0.967)$ and; Perkins and Schisterman (AUC $=0.92$ ) for the PG-SGA tool classified according to SGAC. ${ }^{[18,19]}$ However, PG-SGA tool classified as per SNTR had AUC $=1.0$ with higher accuracy and effectiveness. Therefore, usage of PG-SGA tool classified as per SNTR is better than PG-SGA tool classified as per SGAC.

\section{Conclusions}

Cancer treatment is associated with various NIS. In this study, the severity of NIS was higher in HNC which affected the food intake due to the presence of tumor and treatment, especially due to localized radiotherapy which caused dry mouth, altered taste, mucositis, trismus, etc. However, GIC subjects had symptoms such as nausea and early satiety and; subjects with OC had pain as the main symptom. In general, dry mouth and altered taste perception were present in all cancers. The presence of cachexia along with NIS due to treatment greatly affected the functional status of all cancer patients which was worse in the case of HNC. Nutritional status is important in the case of NIS management. Weight loss which is commonly observed in all cancers is primarily due to decreased food intake, increased catabolism, and increased energy demand by the body, tumor-host competition for nutrients, or stage of cancer. Presence of cancer cachexia will further aggravate the weight loss trajectory. The results of AUC showed near to perfect discrimination between malnourished and well-nourished group as per SGAC. However, the AUC of SNTR was higher with perfect discrimination between nutritional risk groups indicating the use of SNTR as better approach for assessing nutritional status, since SGAC is subjective in nature. Overall, participants with HNC and GIC were affected in functional and nutritional status, severity of NIS, and nutrition risk than OC. The scored PG-SGA is a quick, valid, and reliable nutrition assessment tool than SGA which helps in identifying nutritional risk and developing a better nutritional care plan. Therefore, a planned nutritional intervention based on the PG-SGA data will delay the rate of catabolism and helps in improving the nutritional status, thus promoting prognosis.

\section{Acknowledgment}

We thank Dr. Anil Thomas, Preethi Cancer Centre, Lakshmipuram, Mysuru, Karnataka, India, Dr. Sathya. M and Dr. Mukesh S, Krishna Rajendra Hospital, Sayyaji Rao Road, Mysuru, Karnataka, India, and Dr. Vishweshwara M.S,
HCG-Bharath Hospital and Institute of Oncology, Hebbal, Mysuru, Karnataka, India, for extending their full support in carrying out the study.

\section{Financial support and sponsorship}

This study received financial support from University Grants Commission, New Delhi, under SAP scheme at the level of Departmental Research Support. Grant number: UGC No. F 640/1/DRS/2013 (SAP-I), dated July 15, 2013.

\section{Conflicts of interest}

There are no conflicts of interest.

\section{REFERENCES}

1. International Agency for Research on Cancer. GLOBOCAN; Estimated Incidence, Mortality and Prevalence Worldwide in 2012. International Agency for Research on Cancer; 2014. Available from: http://www. globocan.iarc.fr/Pages/fact_sheets_cancer.aspx. [Last accessed on 2014 Jun 12].

2. World Health Organization-Non communicable Diseases (NCD) Country Profile for India; 2014. Available from: http://www.who.int/ countries/ind/en/. [Last accessed on 2014 Jun 12].

3. Ravasco P, Monteiro Grillo I, Camilo M. Cancer wasting and quality of life react to early individualized nutritional counselling! Clin Nutr 2007;26:7-15.

4. Ottery FD. Patient-generated subjective global assessment. In: McCallum PD, Polisena CG, editors. The Clinical Guide to Oncology Nutrition. Chicago: The American Dietetic Association; 2000. p. 11-23.

5. Ottery FD. Available from: http://www.pt-global.org/?page_id=6098. [Last accessed on 2014 Aug 19].

6. Jager-Wittenaar H, Ottery FD. Assessing nutritional status in cancer: Role of the patient-generated subjective global assessment. Curr Opin Clin Nutr Metab Care 2017;20:322-9.

7. Vigano AL, di Tomasso J, Kilgour RD, Trutschnigg B, Lucar E, Morais JA, et al. The abridged patient-generated subjective global assessment is a useful tool for early detection and characterization of cancer cachexia. J Acad Nutr Diet 2014;114:1088-98.

8. Isenring E, Cross G, Daniels L, Kellett E, Koczwara B. Validity of the malnutrition screening tool as an effective predictor of nutritional risk in oncology outpatients receiving chemotherapy. Support Care Cancer 2006;14:1152-6.

9. Zweig MH, Campbell G. Receiver-operating characteristic (ROC) plots: A fundamental evaluation tool in clinical medicine. Clin Chem 1993;39:561-77.

10. Kubrak C, Olson K, Jha N, Jensen L, McCargar L, Seikaly H, et al. Nutrition impact symptoms: Key determinants of reduced dietary intake, weight loss, and reduced functional capacity of patients with head and neck cancer before treatment. Head Neck 2010;32:290-300.

11. Grant MM, Rivera LM. Anorexia, cachexia, and dysphagia: The symptom experience. Semin Oncol Nurs 1995;11:266-71.

12. Paillaud E, Bories PN, Aita SL, Scherman E, Jeanfaivre V, Lejonc JL, et al. Prognostic value of dietary intake and inflammation on survival in patients with advanced cancer: Relationship with performance status, pain, and digestive disorders. Nutr Cancer 2003;45:30-5.

13. Feuz A, Rapin CH. An observational study of the role of pain control and food adaptation of elderly patients with terminal cancer. J Am Diet Assoc 1994;94:767-70.

14. Omlin A, Blum D, Wierecky J, Haile SR, Ottery FD, Strasser F, et al. Nutrition impact symptoms in advanced cancer patients: Frequency and specific interventions, a case-control study. J Cachexia Sarcopenia Muscle 2013;4:55-61.

15. Theologides A. Weight loss in cancer patients. CA Cancer J Clin 1977;27:205-8.

16. Fearon KC, Voss AC, Hustead DS; Cancer Cachexia Study Group. Definition of cancer cachexia: Effect of weight loss, reduced food intake, and systemic inflammation on functional status and prognosis. Am J Clin Nutr 2006;83:1345-50. 
Shivanna, et al.: Validation of assessment categories of the PG-SGA tool

17. Bauer J, Capra S, Ferguson M. Use of the scored patient-generated subjective global assessment (PG-SGA) as a nutrition assessment tool in patients with cancer. Eur J Clin Nutr 2002;56:779-85.

18. Gabrielson DK, Scaffidi D, Leung E, Stoyanoff L, Robinson J, Nisenbaum R, et al. Use of an abridged scored patient-generated subjective global assessment (abPG-SGA) as a nutritional screening tool for cancer patients in an outpatient setting. Nutr Cancer 2013;65:234-9.

19. Perkins NJ, Schisterman EF. The inconsistency of "optimal" cutpoints obtained using two criteria based on the receiver operating characteristic curve. Am J Epidemiol 2006;163:670-5. 